\title{
The effect of grinding media on the flotation of fine chalcopyrite
}

\author{
Yong Chen ${ }^{1, a^{*}}$, Yong Sheng Song ${ }^{2, b}$, Wen Juan $\mathrm{Li}^{3, \mathrm{c}}$ and Gui Ying Zhou ${ }^{4, d}$ \\ ${ }^{1}$ No.2 Xinjiekouwai Street, Beijing, China \\ ${ }^{2}$ No. 2 Xinjiekouwai Street, Beijing, China \\ ${ }^{3}$ No. 2 Xinjiekouwai Street, Beijing, China \\ ${ }^{4}$ No.2 Xinjiekouwai Street, Beijing, China \\ a cy040311@163.com, bysmba@163.com, c juanzi88888@126.com, \\ dzhouguiying2001@163.com
}

Keywords: fine chalcopyrite, grinding media, flotation, pulp potential

Abstract. The pulp potential and flotation behaviour of fine chalcopyrite ground with different grinding media have been studied by experiments. It's shown that there exist great differences in pulp potential, oxidation species and flotation behaviour of chalcopyrite ground with ceramic or iron media. Grinding with ceramic media gave much higher pulp potential than grinding with iron media, which is much more beneficial to flotation of chalcopyrite. Low flotation recovery of chalcopyrite ground with iron media are mainly due to the surface of chalcopyrite coating with a hydrophilic film which composed of a plenty of $\mathrm{FeOOH}$.

\section{Introduction}

Copper is an important raw material, which mainly has been produced by dry metallurgy at present. Along with the development of new flush smelting technology in industry, the quality of copper concentrate become more important. The flush smelting technology requires copper content of smelting feed be high. So how to effectively separate chalcopyrite from ores becomes an important task in flotation study. As a necessary preparation before flotation, grinding shows great significance for flotation. Grinding, as a kind of complex physical, chemical and physicochemical process, would affect the subsequent sulfide processing considerably[1,2]. Therefore, it has great importance on theory and practice to study the relationship between grinding environment and sulfide flotation.

It is found that there exists intimate relationship among chemical properties of slurry $(\mathrm{pH}, \mathrm{Eh}$, dissolved oxygen content and ion concentration), surface properties of sulfide minerals and grinding environment $[3,4]$. Grinding in different media leads to different chemical properties of slurry and different surface properties of individual sulfide mineral and the dual mineral systems.

In this paper, the pulp potential, the deposite time and the flotation rate of chalcopyrite with different grinding medium are studied. The aim of this work is to obtain the effects of different grinding environment on the floatability of chalcopyrite and explore the effecting mechanism of grinding environment on floatability of chalcopyrite.

\section{Materials and methods}

Samples and reagents

Pure handpicked mineral samples of chalcopyrite were obtained from Del copper mine of Qinhai Province. The mineral sample was crushed, handpicked and dry-ground with a porcelain ball mill and dry-sieved to obtain different size fractions. The fraction less than $74 \mu \mathrm{m}$ was used for flotation studies. The fraction over than $106 \mu \mathrm{m}$ was used for grinding studies. The minerals were stored in a desiccator under nitrogen atmosphere. The purity of the mineral samples was ascertained by mineralogical studies. Chemical analysis indicated that chalcopyrite purity was $92.65 \%$. 
The collector was ethyl xanthate, which was industrial grade product from Chemical Factory of Zhuzhou, China. $2^{\#}$ oil was employed as a frother, which was also industrial grade product. $\mathrm{HCl}$ and $\mathrm{NaOH}$ were adopted to adjust the pulp $\mathrm{pH}$ value.

Grinding test

$20 \mathrm{~g}$ sample was ground in a laboratory stainless steel or ceramic ball mill at $60 \%$ solids to obtain $80 \%$ particles passing $38 \mu \mathrm{m}$. The mill discharge was then transferred to a glass beakers for clarification. The pulp potential was measured after 2 minute.

Flotation test

The flotation tests were carried out in a micro flotation cell with a $40 \mathrm{~mL}$ effective volume under the condition of $\mathrm{pH}=8$, ethyl xanthate $20 \mathrm{~g} / \mathrm{t}$ and $2^{\#}$ oil $10 \mathrm{~g} / \mathrm{t}$. The amount of sample used for each experiment was $2 \mathrm{~g}$, which was ultrasonically washed for $5 \mathrm{~min}$ to remove any possible oxides on the mineral surface. The washing solution was decanted and a fresh solution with a given $\mathrm{pH}$ was added before flotation. The flotation time was $4 \mathrm{~min}$. The flotation recovery $(\mathrm{R})$ was calculated from $\mathrm{R}=\left[\mathrm{m}_{1} /\left(\mathrm{m}_{1}+\mathrm{m}_{2}\right)\right] \times 100 \%$, where $\mathrm{m}_{1}$ and $\mathrm{m}_{2}$ are the mass of the floated and unfloated products, respectively.

\section{Results and discussions}

When the sulfide ore slurry exposing to air for a long time, the slurry properties will change, causing an impact on mineral flotation. The effects of deposited time on pulp potential of chalcopyrite with different grinding media was shown in Fig 1. It demonstrates that the pulp potential of chalcopyrite with different grinding media both declines in the beginning of deposite time $(0 \sim 2 h)$. But with the extension of deposite time, chalcopyrite pulp potential ground with ceramic media basically stable at $380 \mathrm{mv}$, while chalcopyrite pulp potential ground with iron media is only about $345 \mathrm{mv}$.

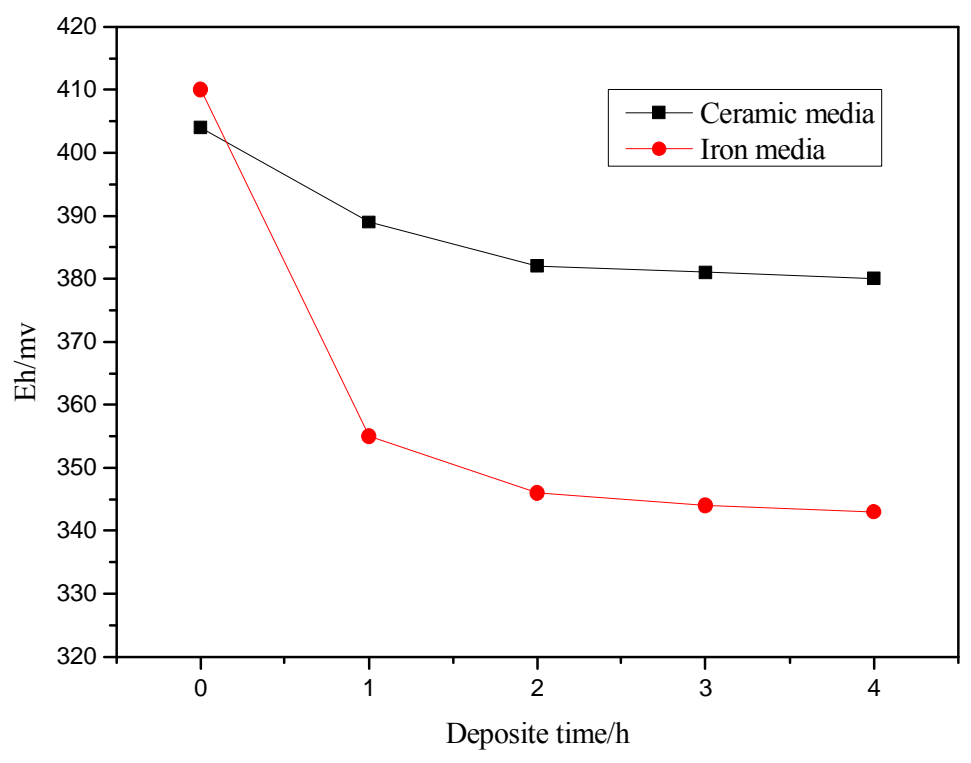

Fig. 1 Effects of deposited time on Eh in pulp of chalcopyrite

Fig. 2 was show the effects of deposited time on flotation of chalcopyrite with different grinding media. The recovery of chalcopyrite basically does not change with the deposite time. The recovery of chalcopyrite ground with iron media was lower than the ceramic media. The reason was that chalcopyrite flotation was strongly dependent on both iron oxidation species and metal deficiency on the chalcopyrite surface. Iron oxidation species from grinding media played a dominant role in depressing chalcopyrite flotation, while metal deficiency from chalcopyrite oxidation improved 
chalcopyrite flotation[5]. Therefore, ceramic grinding medium produced a higher chalcopyrite recovery than iron grinding medium.

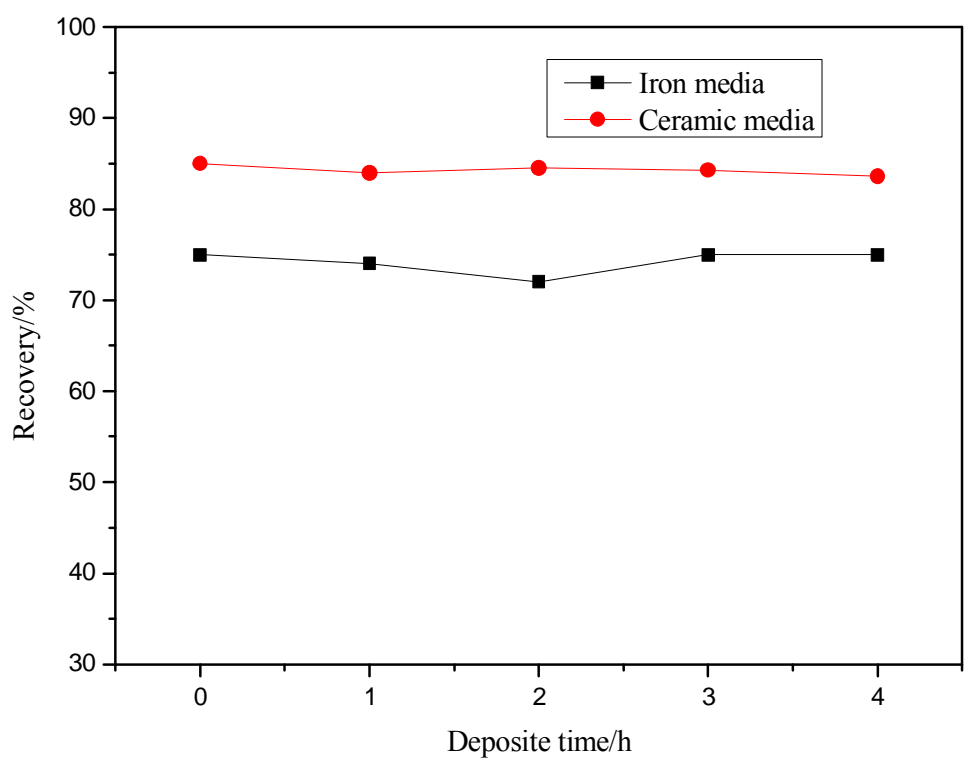

Fig.2 Effects of deposited time on flotation of chalcopyrite

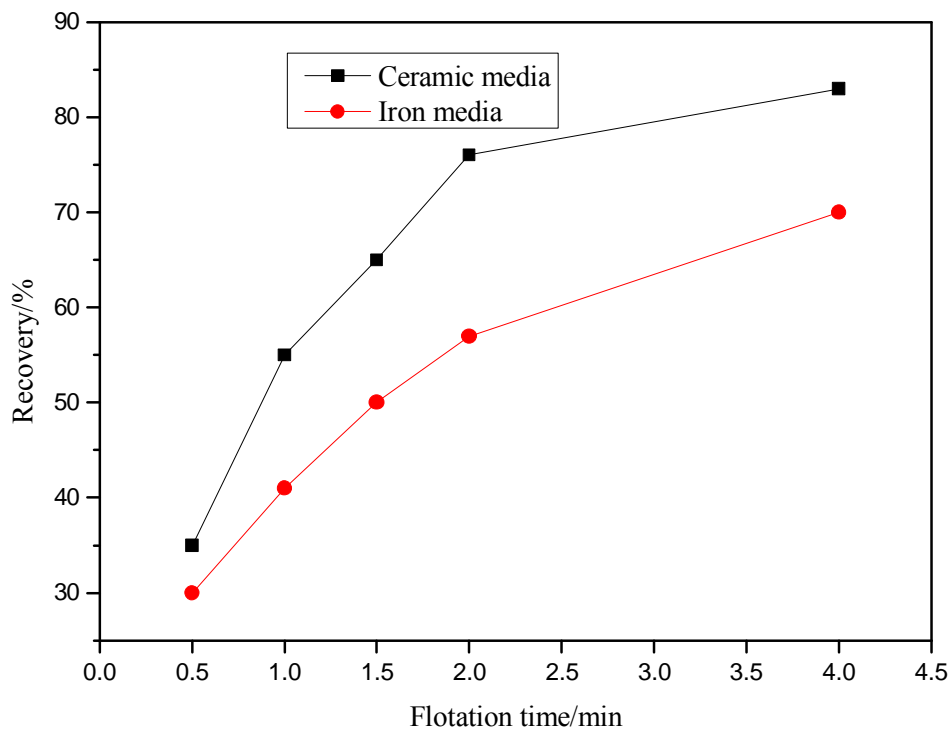

Fig.3 Effects of grinding environment on flotation rate of chalcopyrite

Comparison of the flotation rate of chalcopyrite with different grinding medias as shown in Fig.3. Compared being ground with ceramic media, the chalcopyrite ground with iron media shows approximately the same recovery of copper ( about 30\%) at the beginning of flotation. With the extension of flotation time, the recovery of chalcopyrite ground with ceramic media increased rapidly in 2 minutes. So as the same flotation results of chalcopyrite ground with iron media, but the recovery and flotation speed were much lower than with ceramic media.

The main reasons of causing evident difference on flotation behaviour of chalcopyrite ground with iron or ceramic media are following[6]. Firstly, the deposition and adsorption of hydroxy iron complex on the surface of chalcopyrite would enhance the surface hydrophilicity and reduce the flotability of 
chalcopyrite. Secondly, strong reducing environment caused by ground with iron media would block the moderate surface oxidization of chalcopyrite, which is necessary for flotation, while oxidizing environment caused by ground with ceramic media would be beneficial to the moderate surface oxidization of sulfide minerals. Thirdly, chalcopyrite ground with ceramic media would form a metal-lacking while sulfur-exceeding surface, which would promote flotation of sulfide minerals. Lastly, when ceramic is used as grinding media, the oxidizing environment with higher potential would promote the oxidization of xanthate to dixanthogen, but when iron media is used, the situation would be reversed.

\section{Conclusion}

Through the pulp potential and flotation behaviour of fine chalcopyrite ground with different grinding media test on the sample, it is indicated that great differences in pulp potential, oxidation species and flotation behaviour of chalcopyrite ground with ceramic or iron media. The recovery and pulp potential of fine chalcopyrite ground with ceramic media are both much high than with iron media. The reason was that iron oxidation species from grinding media played a dominant role in depressing chalcopyrite flotation.

\section{Acknowledgements}

The authors acknowledge the Enterprise Technology Innovation Fund project of Qinghai provincial Science and Technology Department (No. 2015-GX-Q06A) for the financial support, and we would like to express our sincere appreciation to the reviewers for their insightful comments, which have greatly aided us in improving the quality of the paper.

\section{References}

[1] Bauer, M., Craig, I.K., Tolsma, E., de Beer, H., 2007. I Industrial and Engineering Chemistry Research 46, 5614-5623.

[2] Craig, I.K., Macleod, I.M., 1995. Control Engineering Practice 3 (5), 621-630.

[3] Herbst, J.A., Alba, J.F., Pate, W.T., Oblad, A.E., 1988. International Journal of Mineral Processing 22, 275-296.

[4] Hulbert, D.G., Craig, I.K., Coetzee, M.L., Tudor, D.,1990. Journal of the South African Institute of Mining and Metallurgy 90 (7), 173-181.

[5] Lo, Y.C., Oblad, A.E., Herbst, J.A.,1996. Minerals and Metallurgical Processing 8, 19-21

[6] Radhakrishnan, V.R., 1999. Journal of Process Control 9 (3), 195-211. 